\title{
Nonlinear Mode Coupling Analysis in the Tevatron
}

\author{
S. Assadi and C.S. Mishra \\ Fermi National Accelerator Laboratory \\ P.O. Box 500, Batavia, Illinois 60510
}

August 1995

Proceedings of 1995 Particle Accelerator Conference and International Conference on High-Energy Accelerators, Dallas, Texas, May 1995. 


\section{Disclaimer}

This report was prepared as an account of work sponsored by an agency of the United States Government. Neither the United States Government nor any agency thereof, nor any of their employees, makes any warranty, express or implied, or assumes any legal liability or responsibility for the accuracy, completeness, or usefulness of any information, apparatus, product, or process disclosed, or represents that its use would not infringe privately owned rights. Reference herein to any specific commercial product, process, or service by trade name, trademark, manufacturer, or otherwise, does not necessarily constitute or imply its endorsement, recommendation, or favoring by the United States Government or any agency thereof. The views and opinions of authors expressed herein do not necessarily state or reflect those of the United States Government or any agency thereof. 


\title{
NONLINEAR MODE COUPLING ANALYSIS IN THE TEVATRON ${ }^{1}$
}

\author{
S. Assadi and C.S. Mishra, Fermi National Accelerator Laboratory, Batavia, IL -60510, USA.
}

Increased demand for higher luminosities from Fermilab proton-antiproton collider requires higher intensities and increased number of bunches. The performance of existing $6 \times 6$ and the proposed $36 \times 36$ collider is often limited by the non-linear effects arising from beam-beam interactions, in particular for high intensity beams. The tune shifts and spread from increased long range interactions combined with higher order multipoles complicates the frequency spectrum of the particle motions. TEAPOT simulation program is used to generate turn by turn data that could be used in the nonlinear mode spectrums analysis. Bispectral mode analysis is discussed.

\section{INTRODUCTION}

A ubiquitous feature of high luminosity Tevatron proton-antiproton collider is the presence of nonlinear forces composed of a spectrum of frequencies and wavenumbers (i.e. higher order multipoles sampled by the particles with the largest beam-beam tune shift). Understanding the spatial and temporal structure of the beam dynamics in connection with chromaticity, the dispersion in RF cavities and higher order multipoles remains a daunting goal of nonlinear beam physics. Nonlinear interactions between spatial Fourier components are the key determinant of the wave vector $(\mathbf{k})$ spectrum of the fluctuating particles.

The analytic theory of synchro-betatron motions was first studied in connection with chromaticity. The dispersion in RF cavities was found to cause beam loss by coupling, and this effect has been studies analytically and computationally by a number of physicist. However, no experimental evidence of considerable wave-wave coupling of the broadband turbulence has been reported so far. Whereas the standard linear spectral analysis ( power spectrum) provides experimental information on the amplitude and phase behavior of the individual Fourier components and the transport induced

\footnotetext{
${ }^{1}$ Operated by the University Research Assoc.

Inc., under contract with U.S. Dept. of Energy.
} 
by fluctuations, it does not give any information about the coupling among different spectral components. The usc of the bispectral analysis allows one to discriminate between oscillations spontaneously excited by the beam and those generated by nonlinear coupling.

The purpose of this paper is to introduce a new analysis tool for turbulent or chaotic data to the accelerator physics community. We investigate the combined effect of long range interactions and higher order multipoles to calculate the nonlinearly generated frequency spectrum. In this paper we discuss three cases. First we restrict ourselves to syncro-betatron and betatron-betatron coupling of a single beam with betatron tunes close to $7 / 12$ resonance when higher order multipoles are present. Second, beam-beam without error fields are considered. Finally beam-beam with multipoles are analyzed. A modified version of thin element tracking program TEAPOT (see paper by C.S. Mishra et al. In these proceedings) has been used to simulate the turn by turn data for Tevatron lattice.

\section{ANALYSIS}

The bicoherence spectrum has been computed to study the strength of the three-wave coupling contributing to the characteristics of beam-beam coupling in the Tevatron.

The bicoherency is defined as

$$
b^{2}\left(v_{1}, v_{2}\right)=B\left(v_{1}, v_{2}\right)^{2} /\left[<\left|X_{v_{1}} X_{v_{2}}\right|^{2}>P(v)\right]
$$

where $\mathrm{B}\left(v_{1}, v_{2}\right)$ is the bispectrum defined as $\mathrm{B}\left(v_{1}, v_{2}\right)=\left\langle\mathrm{X} v_{1} \mathrm{X}_{v_{2}} \mathrm{X} v *>, \mathrm{P}(v)\right.$ the autopower spectrum $\mathrm{P}(v)=\left\langle\mathrm{X} v_{1} \mathrm{X} v 2^{*}\right\rangle, \mathrm{X} v$ is the Fourier transform of the turn by turn data, $\mathrm{X}(\mathrm{t})$ and $\diamond$ means ensemble averaging over many statistically similar realizations. The bicoherency measures the fraction of the fluctuating power at a frequency $v$ which is phase correlated with the spectral components at frequency $v_{1}$ and $v_{2}$ obeying the summation rule $v=v_{1}+v_{2}$. The bicoherency thus quantifies the degree of coupling among three waves. The bicoherency is bound between 0 and 1 : When $b^{2}\left(v_{1}, v_{2}\right)$ is equal to 1 then the oscillations at frequency $v$ are completely coupled with frequency components at frequency $v_{1}$ and $v_{2}$ and completely decoupled for the zero value. The use of the bicoherence as a measure of three-wave coupling is independent of any closure assumptions. 
The bicoherence is nonzero if there is a statistically significant phase relation between the three modes. Such bispectral analysis has been advanced by Ritz, Powers, and Bengston for plasma research, and applied in the frequency domain to electrostatic fluctuations [1].

\section{SIMULATION RESULTS AND CONCLUSIONS}

In order to predict a particle's behavior in the presence of the beam-beam interaction in the Tevatron, modified TEAPOT simulation code was run under three conditions. The predicted position and phase of a particle after a designated 20000 turns is used to calculate the bicoherencies. In all cases, the base tunes are $(\mathrm{Qx}, \mathrm{Qy})=(20.5836,20.5826)$. The two sextupole families are used to set the chromaticity of the Tevatron to a desired value (minimum chromaticities to keep the beam stable at $900 \mathrm{Gev})$; the chromaticity setting is adjusted to $\xi \mathrm{x}=20$ and $\xi \mathrm{y}=24$. The launched particle has $\Delta \mathrm{P} / \mathrm{p}$ $=0.0001$. Beam intensity is $3.0 \mathrm{E} 11$. The initial position and phase is the same for all cases. Figure one shows a small section of the turn by turn data used to calculate the bicoherency. In a first approximation, when the intensity of the opposite beam is relatively low and the tunes are far enough from the low order resonances, the beam-beam effects appear as a nonlinear dependence of the frequencies of transverse oscillations on their amplitude. For larger intensity, or when the tune are close to the low-order resonances, isolated nonlinear resonances, creating a periodic energy exchange between the transverse planes, come into play. This is the regime in which we have performed the simulations. For still larger intensity, the resonances can overlap to form stochastic regions where the particle dynamic is diffusive.

Figure 2 shows the normalized phase space plot for the single beam. The only source of nonlinearites is the higher order multipoles. The above standard normalized phase space plot does not show any structure that could reveal the sources of nonlinearities if any exist. However, Figure 3 shows the calculated bicoherence which clearly shows the three wave coupling. Peak " $\mathrm{A}$ " is the only significant nonlinearity observed which is due to the dispersion present in the cavities. This synchrobetatron coupling can be removed from the simulation by removing dispersion. Figure 4 shows the 

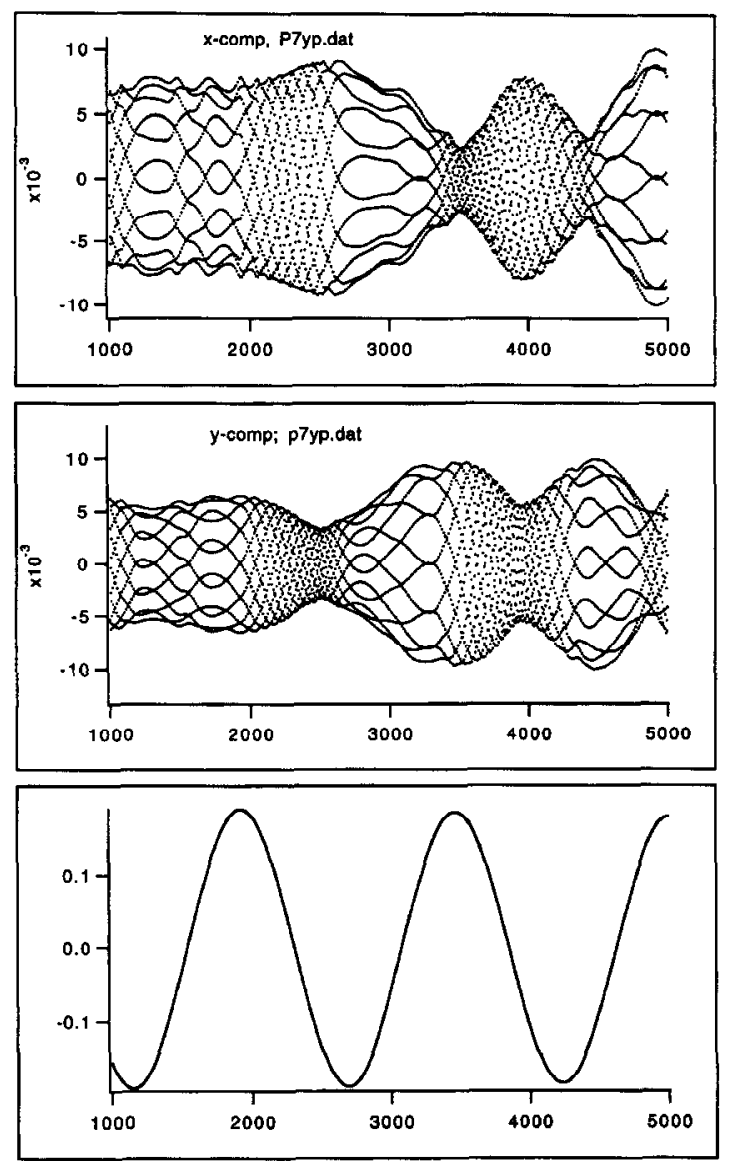

Figure 1.) Small section of the turn by turn plots of a particle with beam-beam is shown. Higher order multipoles are not present. The top plot is the horizontal, the middle plot is the vertical and the lower plot is the synchrotron motion. Clearly the energy exchange between the horizontal and vertical plane during the rise time of the synchronous oscillations is different from the fall.
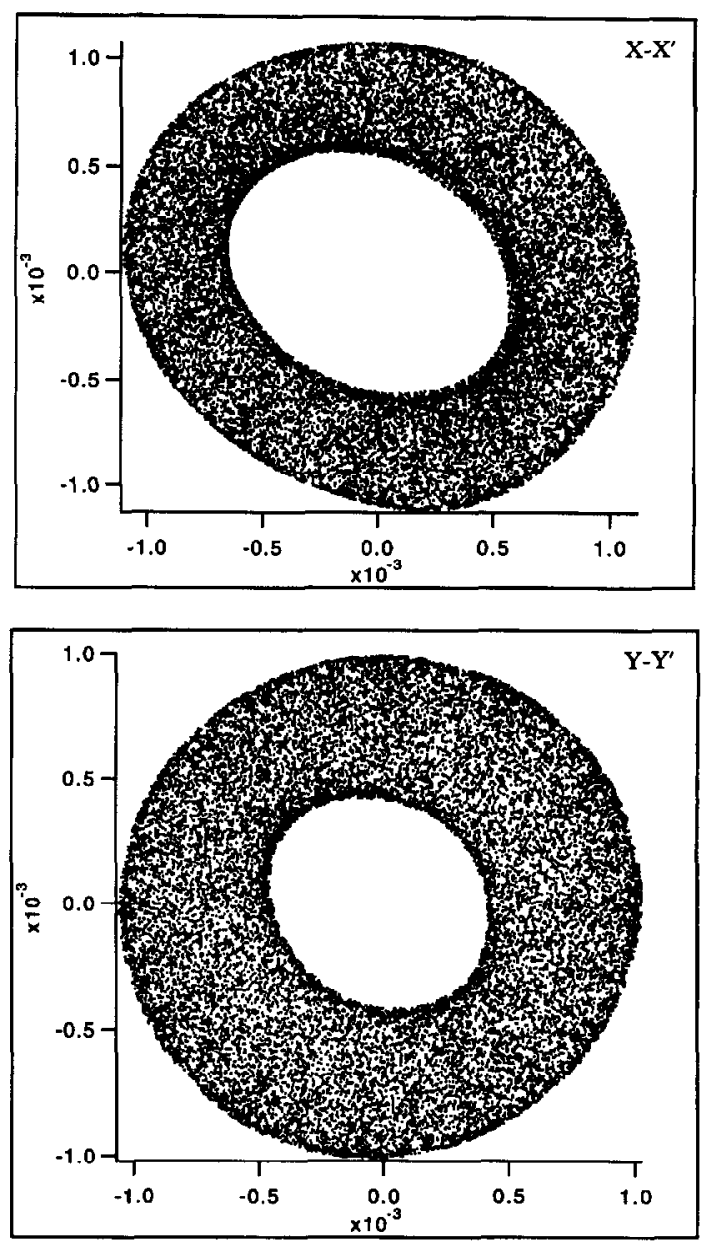

Figure 2). Single beam normalized phase space plots with higher order multipoles is shown. 


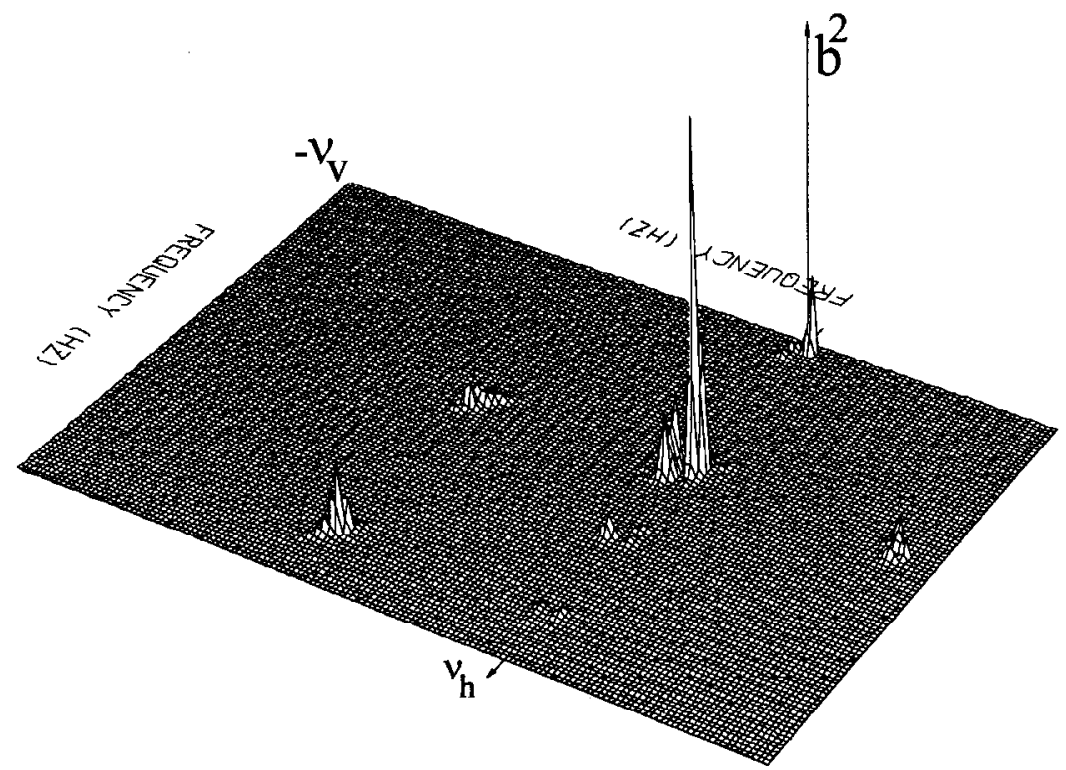

Figure 3) Bicoherence, $b(v 1, v 2, v 3)$, of single beam with all higher order modes present. The horizontal axes denote horiz. and vertical tunes. The vertical axis denotes the bicoherence for $v 3=v 1+v 2$. The larger the amplitude, the stronger the nonlinear coupling is.

normalized phase-space plot for the beam-beam with the long range kicks. The higher order modes are not present. Twelve islands associated with 12 parasitic crossing is observable. This non-linearity is small for small amplitude particles. Figure 5 represent the bicoherence spectrum of beam-beam without the HOM. We observe nonlinearly generated modes due to dispersion and also along the difference resonance $v_{\mathrm{H}}-v_{\mathrm{v}}$. Fig. 6 and Fig. 7 shows the normalized phase space as higher order multipoles added to the case 2 of Fig. 4 and Fig 5.

\section{REFERENCE}

1) Ch. Ritz, et al., Phys. Fluids B I, 153 (1989). 

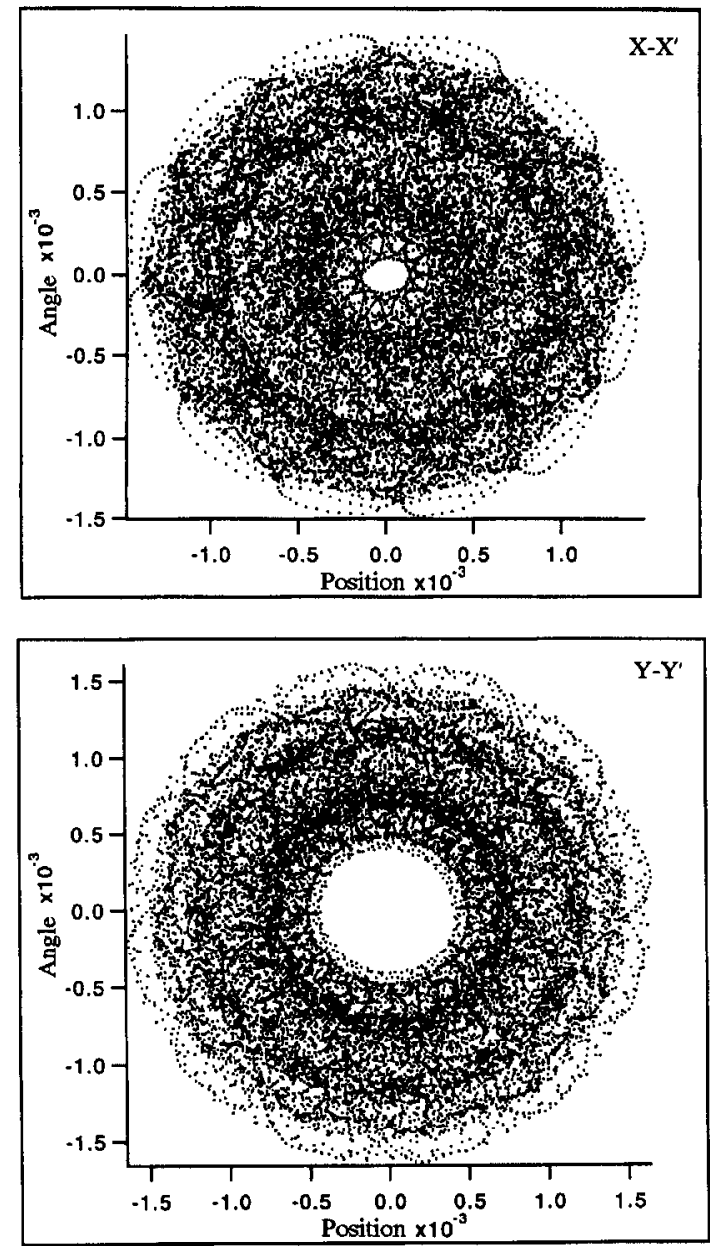

Figure 4). Normalized phase-space plots for beam-beam without higher order multipoles is shown.

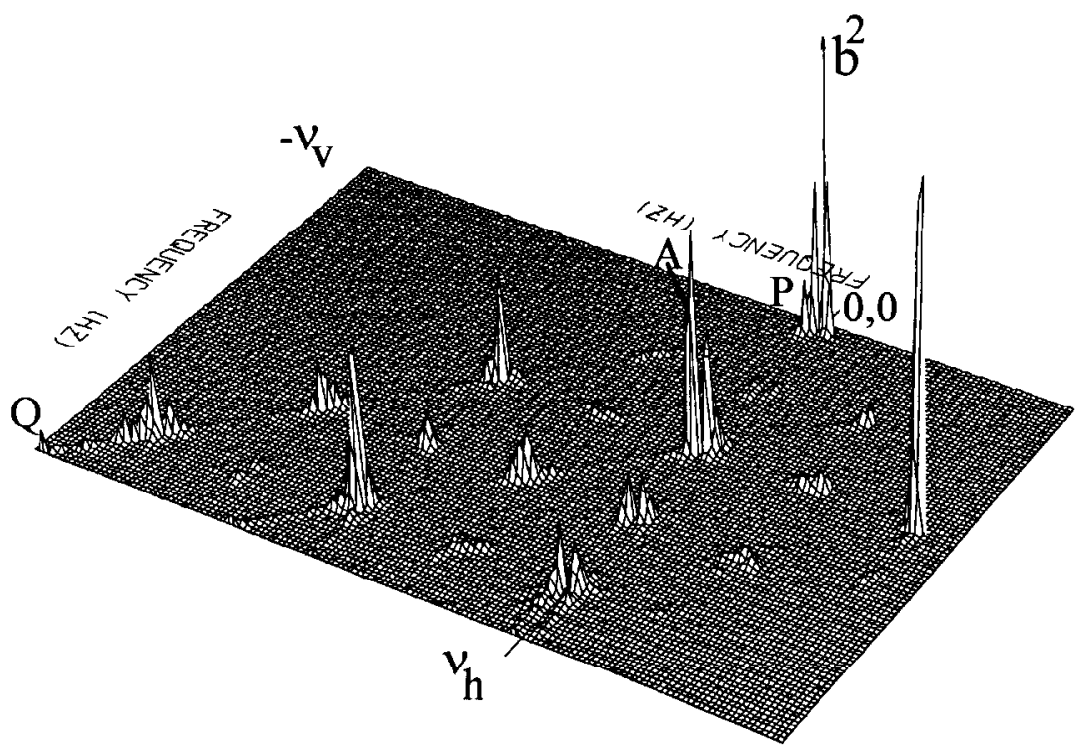

Figure 5). Bicoherence, of beam-beam without HOM. 

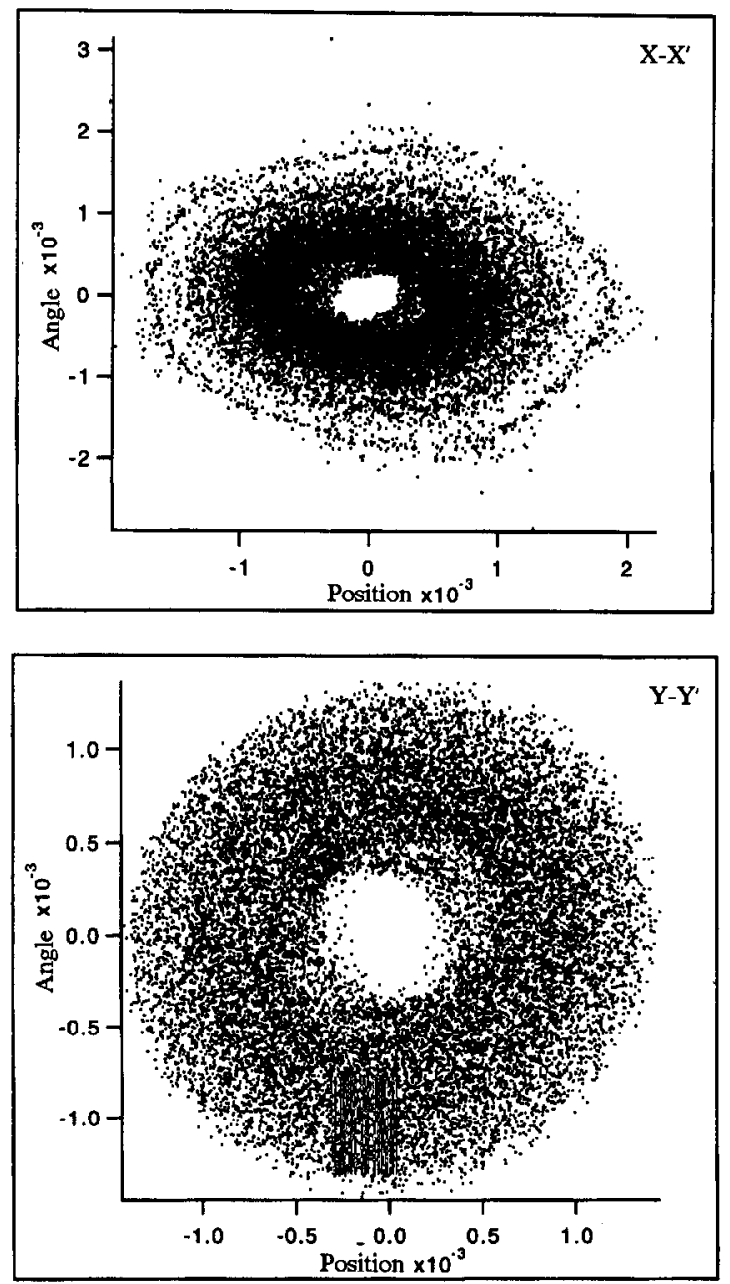

Figure 6). Normalized phase-space plots for beam-beam with higher order multipoles is shown.

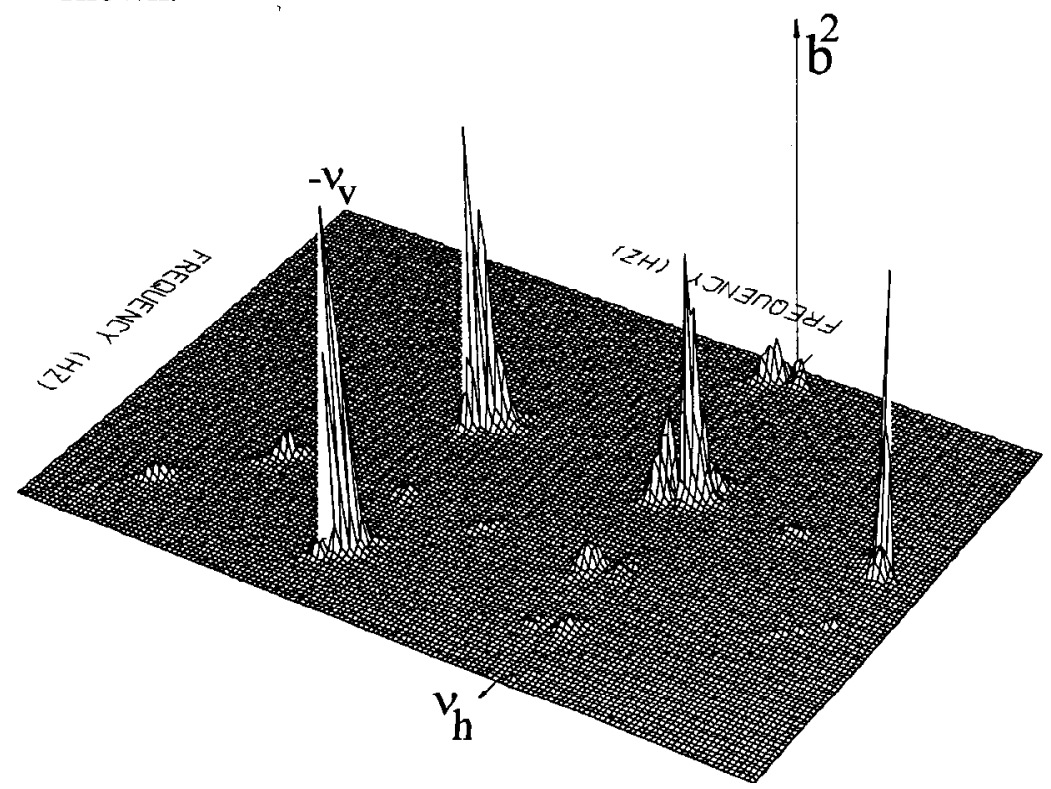

Figure 7) Bicoherence of beam-beam with all higher order multipoles present. 